\title{
Les rêves océaniens de Jacques et Betty Villeminot
}

\section{Christian Coiffier}

\section{(2) OpenEdition}

\section{Journals}

Édition électronique

URL : http://journals.openedition.org/jso/6877

DOI : $10.4000 /$ jso. 6877

ISSN : $1760-7256$

Éditeur

Société des océanistes

Édition imprimée

Date de publication : 15 octobre 2013

Pagination : 233-238

ISBN : 978-2-85430-035-2

ISSN : 0300-953x

Référence électronique

Christian Coiffier, "Les rêves océaniens de Jacques et Betty Villeminot », Journal de la Société des Océanistes [En ligne], 136-137| 2013, mis en ligne le 20 octobre 2013, consulté le 15 avril 2020. URL : http://journals.openedition.org/jso/6877 ; DOI : https://doi.org/10.4000/jso.6877

(C) Tous droits réservés 


\section{MISCELLANÉES}

\section{Les rêves océaniens de Jacques et Betty Villeminot}

par

\section{Christian COIFFIER*}

Jacques et Betty Villeminot sont de fidèles adhérents de notre Société depuis fort longtemps ${ }^{1}$. Ce petit résumé de leurs activités océaniennes me permet de m'acquitter d'une dette profonde que j'ai contractée à leur l'égard, car je leurs dois en partie la passion et l'intérêt que je porte à l'Océanie depuis mon adolescence. C'est la vision de leurs films sur la Nouvelle-Guinée et la lecture de leurs différents ouvrages qui me firent découvrir les prestigieuses architectures du Sepik que j'allais ensuite étudier durant plusieurs années. C'est pourquoi j'ai souhaité en guise de remerciements leur dédier ces quelques lignes. Je me souviens particulièrement que Jacques Villeminot annonça, durant une de ses conférences sur la Nouvelle-Guinée à la fin des années 1960, que toutes les scènes dont il venait de présenter les images ne seraient plus visibles d'ici une dizaine d'années. En fait, je pus constater personnellement une vingtaine d'années plus tard que des pans entiers des cultures du Sepik qu'il avait présentées avaient résisté aux divers changements apportés par l'indépendance du pays.

Après avoir été reporter-photographe et journaliste à Paris, Jacques Villeminot part avec son épouse Betty faire le tour du monde. En 1951, après avoir visité les principales îles de la Polynésie française, il réalise un film documentaire, Visages d'Océanie, qu'il présente au public dans le cadre des conférences «Connaissance du Monde ».

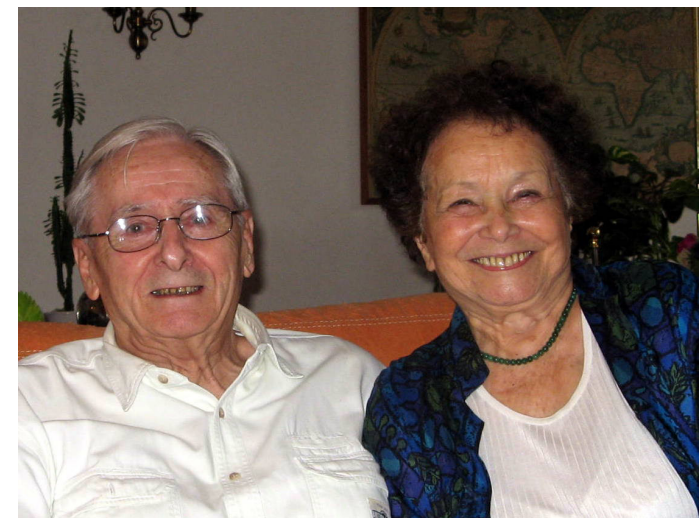

Рното 1. - Jacques et Betty Villeminot (cliché familial)

Le couple part alors vivre en Australie durant près d'une année. Après un bref retour en France, Jacques et Betty Villeminot retournent en 1956 pour huit mois en Australie où ils souhaitent accomplir une mission ethnographique chez les Pitjantjara, un peuple aborigène encore peu connu à l'époque. Ils préparent longuement cette expédition en collaboration avec le professeur A. P. Elkin du département d'anthropologie de l'Université de Sydney. Ils reçoivent le soutien du Muséum national d'histoire naturelle de Paris et de la Société de Géographie, ainsi que celui de l'Université de Sydney. Ils rapportent de cette mission une

1. NDLR. - Jacques, parrainé par le docteur Villaret et Jacques Boullaire, a été élu membre de la Société dans la séance du 23 juillet 1954.

* Maître de conférences du MNHn et chargé de mission au musée du quai Branly, coiffier@mnhn.fr 
très précieuse documentation qui vient conforter le directeur du musée de l'Homme, le professeur Henri Valois, de leur avoir accordé sa confiance. Les résultats des observations ethnographiques et des listes de vocabulaire sont remis à Georges Métais, les mensurations anthropométriques d'une cinquantaine de sujets des deux sexes sont transmises à Raoul Hartweg, les enregistrements de musiques et de chants sont confiés à Gilbert Rouget et une vingtaine d'objets ethnographiques sont offerts au département d'Océanie dirigé par Françoise Girard (J. Villeminot, 2008: 168). Les Villeminot ont recueilli en outre au cours de cette mission trente-deux peintures enfantines et constitué une documentation photographique de mille six cents photos en noir et blanc et en couleur. L'expédition est célébrée par des médias comme Paris-Match et France-Soir et présentée dans le Magazine des Explorateurs de Pierre Sabbagh sur l'unique chaîne de télévision de l'époque. Beaucoup de foyers français découvrent alors l'existence des Aborigènes australiens présentés par le couple Villeminot comme :

« un peuple exceptionnel vivant toujours hors des contraintes de notre monde. » (J.Villeminot, $2008:$ : 168)

Jacques Villeminot tourne différents documentaires en Australie et a la surprise de voir l'un de ceux-ci acheté par le département des Affaires indigènes du gouvernement australien. Ce film sur la vie d'une communauté aborigène séjournant auprès d'un poste administratif comporte des séquences de cérémonies très secrètes. Jacques Villeminot tourne alors un film en $16 \mathrm{~mm}$ couleur, intitulé Paradis primitif, qui présente la vie quotidienne d'une communauté aborigène alors peu connue, les Pitjantjara. Il le présente à Jean Rouch qui le trouve excellent et ne fait pas de remarque particulière. Ce film est donc projeté pour la première fois en février 1958 à la salle Pleyel devant un public parisien de deux mille spectateurs. Deux séances supplémentaires sont nécessaires, ce qui est le prélude à une série de nombreuses conférences à travers la France, la Belgique et le Canada. Paradis primitif est remarqué par le producteur de films «Les Écrans modernes », Fred Tavano, qui propose d'en faire un court métrage en $35 \mathrm{~mm}$ sous le titre Les Hommes oubliés d'Australie. Cette nouvelle version du film est donc projetée sur les Champs-Élysées en première partie de programme. Elle obtient diverses récompenses, remportant le premier prix au festival de courts métrages de Tours et à celui d'Oberhausen, une nomination par le Centre national du Cinéma, puis le prix du meilleur documentaire de l'année 1960 à Londres. Un producteur canadien en fait un second court métrage sous le titre Les Hommes oubliés de la prébistoire pour des chaînes de télévision canadiennes et américaines (J. Villeminot, $2008: 169)$.
Jacques et Betty Villeminot sont venus présenter et commenter leur film Les Hommes oubliés d'Australie le 27 janvier 2011, dans le cadre du cinéma des Océanistes au musée du quai Branly, soit plus de cinquante années après son tournage dans le désert australien. Ce film demande à être vu en tenant compte du contexte de l'époque de sa réalisation. La France était en pleine reconstruction après les destructions de la guerre de 1939-45 et l'Australie était alors une terre très lointaine et bien peu connue des Français, si ce n'est par les jeux Olympiques organisés à Melbourne en novembredécembre 1956. Les voyages aériens et la télévision n'étaient pas encore ce qu'ils sont devenus actuellement. Les conférences « Connaissance du Monde » représentaient alors une fenêtre ouverte sur le monde, ce qui les rendait très populaires tant dans la France métropolitaine qu'Outre-mer. Le film présenté dans la salle de cinéma du musée du quai Branly est une version numérisée de 70 minutes (Production Eurocorp) du film de 1960 intitulé Les hommes oubliés d'Australie. Il présente la vie quotidienne d'un groupe d'Aborigènes Pitjantjara stationnés auprès d'un point d'eau dans le désert australien. Après des images de la première rencontre avec les cinéastes, nous voyons successivement des jeux d'enfants, l'obtention du feu par friction, la récolte de fourmis à miel, l'utilisation du boomerang et du propulseur de flèches, la chasse au kangourou et quelques techniques culinaires. Le film nous fait découvrir également la vie rituelle des Pitjantjara avec les peintures rupestres, l'assemblée des anciens et l'utilisation d'objets associés à des rites secrets. Le documentaire se termine par un grand corroboré au cours duquel les hommes mettent en scène leurs ancêtres totémiques. Les commentaires originaux de Jacques Villeminot sur la bande son n'ont guère vieilli, preuve de la considération et du respect que les deux voyageurs portèrent à ces hommes et femmes du désert australien encore peu habitués aux relations avec les Blancs. L'expression «hommes de l'âge de pierre » est cependant parfois utilisée par le commentateur. Si elle n'est plus guère utilisée de nos jours dans ce contexte, elle ne choquait pas à l'époque car elle correspondait à une pensée évolutionniste encore très répandue dans les sciences humaines. Pourtant, les Pitjantjara vivaient pleinement dans le $\mathrm{Xx}^{\mathrm{e}}$ siècle, mais d'une manière très différente de celle des Occidentaux, en utilisant encore parfois, il est vrai, des techniques de l'âge de pierre. Après la projection du film, Jacques et Betty se prêtèrent au jeu des commentaires. A la question d'une spectatrice leur demandant s'ils étaient retournés sur les lieux du tournage, ils répondirent par la négative car l'occasion ne s'était jamais présentée. Jacques et Betty Villeminot ont cependant ouvert la voie à de nouvelles générations d'ethnologues français, comme Vanessa Castejon, Estelle Castro, Aude Chalon, Jessica De Largy, Laurent Dousset, Lise Garond, Barbara Glowczewski, Géraldine Le Roux, Marika 
Moisseeff, Arnaud Morvan, Martin Préaud, qui continuent aujourd'hui leur œuvre de reconnaissance des cultures aborigènes en Australie.

Cette séance de cinéma fut l'occasion pour ces grands voyageurs de présenter leur dernier ouvrage, préfacé par Yves Coppens, Les hommes oubliés d'Océanie (2010) qui retrace en images leurs différents séjours en Océanie ${ }^{2}$. En effet, après l'Australie, le couple s'intéressa à un territoire encore sous mandat australien à cette époque et situé plus au nord, le Territory of Papua and New-Guinea représentant la moitié est de l'île de NouvelleGuinée. Ils y firent plusieurs expéditions avec, à chaque fois, le tournage d'un film et l'écriture d'un livre. Dans les années soixante, très peu de Français connaissaient l'existence de la NouvelleGuinée, mais le public averti des collectionneurs d'arts extra-européens en avait entendu parler, entre autres, à l'occasion du voyage de La Korrigane largement médiatisé trente ans auparavant. Le couple Villeminot arriva ainsi en 1960 dans la vallée de la Wahgi située dans les hauts plateaux des Highlands. En tant que chargé de mission du Muséum de Paris et de l'Université de Sydney, il obtint l'autorisation de se rendre dans la région de Telefolmin encore classée à cette époque Restricted area, c'est-à-dire interdite aux visiteurs européens. Il était cependant accompagné d'officiers de patrouille australiens.

En 1961, c'est dans un petit avion que Jacques et Betty Villeminot arrivèrent au bord du fleuve Sepik. Ils eurent la chance de trouver une pirogue monoxyle motorisée pour descendre le fleuve jusqu'au lac Chambri car, à cette époque, la majorité des villageois manœuvraient leurs esquifs à l'aide de rames. Les Villeminot visitèrent le village de Kanganaman au début de l'année 1961, soit quelques semaines avant le passage de l'allemand Meinhard Schuster de l'Institut Frobenius. C'est ainsi qu'ils purent admirer et filmer les piliers sculptés de la petite maison cérémonielle Mundjimbit (Villeminot, 2010: 110-111) avant que ces derniers ne soient déposés et transportés au Museum für Völkerkunde de Francfort par Meinhard Schuster (Coiffier, 2010 : 264-265). De nos jours, la grande maison Wolimbi du village de Kanganaman existe toujours, telle que les photos nous la présentent (pp. 108-109), même si elle a été restaurée après le tremblement de terre de 1981 qui l'avait bien endommagée. Elle a été classée monument historique national peu de temps après l'indépendance de l'État de Papouasie-Nouvelle-Guinée en 1975. Lors d'une mission en 1965-1966, Jean Guiart rapportera, quelques années après leur passage, une des sculptures de faitage qui se trouve actuellement exposée au musée du quai Branly (Coiffier, 2009: 228-229).

Il faut noter, dans l'ouvrage Les hommes oubliés d'Océanie quelques petites inexactitudes. Tous les crochets sculptés ne servaient pas à y suspendre des têtes surmodelées et le tabouret d'orateur n'était pas un siège pour s'asseoir mais une sorte de pupitre indispensable pour tous débats oratoires (p. 110). Aujourd'hui encore, les initiations ont lieu dans de grandes maisons construites à cet effet et les hommes soufflent toujours dans de longues flûtes qui demeurent interdites à la vue des femmes. Cependant, hommes et femmes du Sepik utilisent maintenant des téléphones mobiles depuis quatre ans. Sur les bords du lac Chambri, qui n'est pas un bras mort du fleuve Sepik (p. 117), la fabrication des poteries n'a pas cessé mais leurs décorations ont bien changé et les couleurs utilisées proviennent maintenant des magasins chinois de la ville.

Jacques et Betty purent ensuite utiliser la nouvelle piste qui venait d'être tracée pour se rendre en voiture de Pagwi, au bord du fleuve, jusqu'à Maprik, à la découverte des Abelam, cultivateurs d'ignames et bâtisseurs de majestueuses maisons de culte. S'ils n'avaient pas la réputation d'être des coupeurs de tête comme ceux des bords du fleuve Sepik, ils étaient déjà connus pour leurs compétitions agricoles consistant à produire les plus gros et longs ignames possibles. Les compétitions d'ignames existent encore, mais les grandes maisons de culte, les «cathédrales d'écorces » (selon l'expression de Jacques Villeminot) qui pouvaient atteindre plus de vingt mètres de hauteur, n'existent quasiment plus en 2011 (p. 122). L'argent gagné ces dix dernières années grâce à la culture de la vanille et le développement des nouvelles religions évangélistes dans la région ont changé profondément les coutumes locales (Coupaye, 2009). Les grandes façades triangulaires de ces maisons constituées de peintures cousues n'ont jamais été soutenues pas des « murs » en bambou tressé (p. 126) car elles se trouvaient attachées aux divers éléments de la charpente constituant les deux pans de l'édifice. Ces peintures n'étaient pas réalisées sur des écorces mais sur des infrabases de palmes de sagoutier.

Les archives du Muséum national d'histoire naturelle conservent divers courriers échangés entre Jacques Villeminot et le département d'Océanie du musée de l'Homme. Dans le courant du mois de novembre 1960, les Villeminot se trouvaient à Telefolmin dans la région du Haut-Sepik sur le territoire de Papua New Guinea (l'actuelle Papouasie-Nouvelle-Guinée). Ils envoyèrent une lettre à Françoise Girard, conservatrice du département Océanie du musée de l'Homme, pour lui faire savoir qu'ils avaient collecté divers objets pour le musée de l'Homme. Ils proposèrent de faire parvenir cette collection au consulat français de Sydney d'où l'administration du musée de l'Homme pourrait faire le nécessaire pour l'acheminer en France. De nombreux courriers entre Françoise Girard, le consulat de France à Sydney, la compagnie des Messageries maritimes et Jacques Villemi- 
not attestent des difficultés administratives qu'il fallut surmonter pour que cette collection puisse rejoindre les réserves du musée de l'Homme après un voyage sur le navire Ventoux et un séjour plus long que prévu sur les quais de Marseille. Cette collection comporte soixante quinze pièces des Highlands et une dizaine provenant de la région du Sepik. Elle se trouve maintenant conservée au musée du quai Branly (71.1961.1 à 92) avec la vingtaine d'objets provenant d'une première expédition chez les Aborigènes d'Australie, donnés par les Villeminot au musée de l'Homme (71.1957.52.1 à 21).

Une seconde séance du cinéma des Océanistes a été consacrée, le 3 novembre 2011, à la projection d'un second film réalisé par Jacques et Betty Villeminot en 1966. Ces derniers n'ont pas pu être présents pour commenter ce documentaire de 52 minutes, Le paradis perdu des îles Trobriand. Ce dernier, intitulé primitivement Les seigneurs des mers du Sud et numérisé par Anako Productions, dans le cadre de leur collection « Les voix de l'oubli », avec ajout d'une présentation par les auteurs, laisse trop transparaître la nostalgie de ces derniers pour un passé maintenant révolu. Cependant, ce film demeure un document ethnographique de première importance, puisqu'il présente des images de diverses cérémonies d'échanges, comme celles des poteries des îles Amphlett et celles de jupes-monnaies en feuilles de bananier, cérémonies sur lesquelles Bronislav Malinowski n'avait guère focalisé son intérêt.

Avant la publication de leur dernier ouvrage Les hommes oubliés d'Océanie, Jacques et Betty Villeminot avaient, chacun de leur côté, réalisé un livre constituant une sorte de testament reflétant leur différentes personnalités pourtant si complémentaires. Ce n'est donc pas étonnant que chacun de ces livres comporte dans leur titre le mot « rêve » qui est un concept majeur dans les sociétés aborigènes. Ce mot y est associé à la vie et à l'éternité, ce qui résume très bien leurs quêtes personnelles au cours de leurs longues années d'errance en Océanie. Ces deux ouvrages nous montrent que c'est bien en Australie, et particulièrement au contact des communautés aborigènes, qu'ils ont su enfin trouver ce qu'ils recherchaient sur cette terre.

Villeminot Jacques, 2008. Mémoires d'Océanie. Vivre de son rêve, Jouy-sur-Morin, Éditions Pages du monde, coll. Anako-Grands témoins, 240 p. et 24 p. de photos couleur et noir et blanc en deux dossiers.

Ce dernier ouvrage est une sorte de testament dans lequel Jacques Villeminot fait le bilan d'une vie bien remplie en compagnie de son épouse Betty à la découverte de terres océaniennes encore bien peu connues des Français à la fin de la Seconde Guerre mondiale. C'est un livre de souvenirs dont les trois quarts sont dévolus aux aventures tahitienne et australienne des Villemi- not. Les expéditions dans le reste de l'Océanie ne sont abordées qu'à la page 179. Comme, vingt-six ans auparavant, lorsque les deux couples de Ganay et van den Broek partirent sur leur yacht $L a$ Korrigane pour deux années et demie en laissant chacun un enfant en bas à la charge des grands-parents, l'attrait des mers du Sud et la réalisation de leurs rêves d'aventures furent plus forts que la vie familiale pour le couple Villeminot. Une certaine culpabilisation de cet «abandon » est perceptible, de façon récurrente, dans une grande partie de l'ouvrage (pp. 9, 44, 48, 57, 62, 126, 166-167).

Dès les premières pages, nous sommes plongés dans un monde maintenant révolu, lorsqu'il fallait quarante jours de navigation pour atteindre Tahiti. Jacques Villeminot décrit très bien le microcosme des voyageurs installés sur le Sagittaire, un paquebot des Messageries maritimes. Le couple fait la connaissance de diverses personnes dont les noms ne sont pas inconnus des Océanistes comme Gabriel Lingé, Jacqueline et Bernard Villaret ainsi que des membres de la famille Lafleur de Nouméa. Le navire emprunte la route maritime classique qui passe par le canal de Panama, les îles Galapagos et les îles Marquises. Les escales dans ces dernières îles représentent une savoureuse introduction au monde polynésien pour le couple Villeminot avant son arrivée à Tahiti le 25 juillet 1951 . Papeete vivait encore dans l'ambiance de l'après-guerre du Pacifique qui avait vu affluer des milliers de militaires américains et la vie nocturne se concentrait alors autour des bars et dancings à la mode, le Tahiti Hut, le Lafayette, le Col bleu ou le Quinn's. La transformation du port de Papeete en «SaintTropez des mers du Sud » (p. 26) ne se fera qu'une dizaine d'années plus tard avec l'installation du centre expérimental à Mururoa en 1960. Après un tour des côtes et l'ascension de quelques sommets à l'intérieur de l'île de Tahiti, le couple part à la découverte des autres îles de la Société en goélette. Il rencontre d'autres futures personnalités locales comme le futur député Francis Sandfort et le photographe Sylvain. Le développement des pellicules photographiques et le traitement des films tournés représentent un souci constant pour Jacques Villeminot, car de leur réussite dépend le succès de leur expédition. Le couple décide finalement de continuer le voyage vers l'Australie pour réaliser complètement son rêve. Il profite du passage d'un navire britannique, le Chungking, pour partir en direction des Nouvelles-Hébrides, l'actuel Vanuatu. Ils ont la chance d'y faire la connaissance de Madame Anthonioz, l'épouse du représentant de la France à Port-Vila. Ce dernier met à leur disposition une voiture qui leur permet de visiter confortablement l'île d'Éfate. Deux jours plus tard, ils arrivent à Nouméa où ils sont accueillis par M. et Mme Lafleur qui les logent et les aident à découvrir le pays. Le 9 novembre 1951, ils arrivent finalement au bout de leur rêve, en Australie, pays auquel l'auteur promet un grand avenir pour les décennies futures. 
Après des débuts très difficiles à Sydney, le couple s'adapte et, sept mois plus tard, Jacques Villeminot réussit à présenter une série de ses photographies de Polynésie à la galerie Kodak de George Street. Ils sont parmi les premiers Français à découvrir le centre de l'Australie. Ils deviennent amis avec le peintre Karel Kupka, un artiste qui s'intéresse à l'art des Aborigènes. À leur retour en France, PaulÉmile Victor propose la candidature de Jacques Villeminot à la Société des Explorateurs dont il deviendra le président quelques années plus tard (p. 137). Jacques Villeminot suit alors des cours à l'institut d'ethnologie du musée de l'Homme.

Jacques Villeminot a manifestement des talents de conteur car il sait très bien situer géographiquement les pays visités et en présenter sommairement leur histoire. Il est cependant dommage qu'un certain nombre de passages de l'ouvrage soient reproduits sans corrections à partir de ses publications précédentes. Quelques erreurs n'ont pas été corrigées, comme l'île de Vanikoro qui ne se trouve pas aux Nouvelles-Hébrides (l'actuel Vanuatu) mais dans l'archipel des Santa Cruz au sud des îles Salomon (pp. 51 et 67), la proclamation de la colonie britannique australienne de New South Wales en 1788 et non en 1778 (p. 66). L'auteur relate assez rapidement, dans les cinq derniers chapitres de l'ouvrage, ses missions dans diverses régions de Nouvelle-Guinée, toujours accompagné de son épouse Betty, plus de dix ans avant l'indépendance de ce pays. Ils vont filmer et enregistrer des chants dans la vallée de la Waghi (pp. 179-186), pénètrent en zone classée Restricted area (pp. 187-200), puis découvrent le fleuve Sepik et les collines Abelam (pp. 201-212). En 1966, ils partent vers les îles Trobriand sur les traces de B. Malinowski (pp. 213224). Quelques années plus tard, ils réservent leur dernière expédition en Nouvelle-Guinée pour aller filmer le peuple Asmat vivant dans les marécages de la côte des Casuarines dans l'ancienne Nouvelle-Guinée Hollandaise aujourd'hui province indonésienne de West Papua (p. 233). Un cahier de dix pages de photographies couleurs et de six pages de photographies noir et blanc, choisies parmi les plus belles de leurs archives, vient agrémenter ce petit ouvrage que tout amateur de l'Océanie se doit d'avoir lu.

Villeminot Betty, 1997. Alcheringa. Le temps du rêve éternel, Paris, Albin Michel, 308 p.

Betty Villeminot a été de presque tous les voyages de son époux Jacques. Dans cet ouvrage, l'auteur se plaît à jouer avec le temps et les identifications. La profonde connaissance de la sensibilité des Aborigènes australiens que Betty a fréquentés durant des années en compagnie de son époux, Jacques, lui permet de se mettre dans la peau d'un vieil aborigène enfermé dans une prison à la fin du xixe siècle pour le vol d'un veau. Betty Villeminot utilise les même artifices qu'Erich Scheurmann dans son célèbre ouvrage Le Papalagui, publié en 1920, qui décrit la vie des Européens à travers le supposé regard d'un chef samoan nommé Tuiavii. L'auteur présente ainsi un vieil homme qui n'a pas mangé depuis plusieurs jours car il ne peut accepter de consommer la viande de son totem, le chien dingo, qui lui est servi par ses geôliers blancs. Dans son délire dû à la sous-alimentation, il voit différents épisodes de sa vie qui nous font revivre tous les événements tragiques qui émaillèrent l'histoire de la conquête des territoires aborigènes par les hommes blancs. Il se remémore ainsi successivement l'arrivée des premiers bateaux, des premiers hommes blancs, des premiers chevaux, des bœufs, des premiers massacres perpétrés par les colons blancs à l'aide de bizarres bâtons d'où sortait un feu destructeur, la vision du premier prêtre évangélisateur dont la soutane noire le fait ressembler à un grand oiseau noir de mauvais augure, l'étrange bête bosselée qui n'était qu'un chameau, bête de trait de l'homme d'Église. L'homme se souvient des incompréhensions des siens vis-à-vis des idées chrétiennes, de la perte de son pouvoir d'homme-médecine lorsque le prêtre arriva à guérir une femme à l'aide de poudre magique. Il se souvient du jour où le prêtre a mis le feu aux écorces peintes considérées comme des objets sacrés et quand des hommes sont venus profaner des lieux sacrés et voler des tjuringa. Il n'a pas oublié le jour où le prêtre a échangé une jeune femme contre des outils de métal pour la transformer en religieuse, l'envahissement des produits commerciaux apportés par les Blancs et les couvertures volontairement infestées, les épidémies de variole qui s'en suivirent avec la pollution volontaire des points d'eau qui amenèrent leur cortège de morts dans tous le pays, les nombreux viols de ses sœurs perpétrés par les Blancs avinés, le développement de l'alcoolisme, les policiers qui jettent ses frères en prison. Il voit ses frères essayer les messes protestantes, catholiques ou baptistes, les forêts d'eucalyptus qui disparaissent devant l'accroissement du nombre de bovins et d'ovins, les ravages des lapins, la cupidité des Blancs pour un métal jaune, l'or. Il se rappelle les luttes vaines en compagnie de ses frères pour essayer de stopper l'avancée des envahisseurs dont la supériorité des armes n'avait d'égale que leur cynisme. Le texte met bien en évidence la totale incompréhension entre des êtres qui, les uns comme les autres, se considéraient comme des sauvages, des non humains. Il a le présage de ce qui va se dérouler dans les années à venir, comme l'irradiation de certaines communautés lors d'essais nucléaires (p. 303) ${ }^{3}$. Alors devant un futur qui lui semble totalement vain, avec des épisodes à venir encore plus sombres que ce qu'il a 
connu, le vieil homme décide de mettre fin à ses jours par pendaison. Cet ouvrage est empreint d'un grand pessimisme, mais il est basé sur une réalité qui fait froid dans le dos. Un lexique des termes Aborigènes utilisés dans le texte clôt cet ouvrage. Ce livre a été traduit en espagnol sous le titre El tiempo del Sueno eterno (B.S.E. Barcelona, 1998), il a été publié également deux fois en anglais sous les titres Alcheringa (Duffy and Snellgrove, Sydney, 2004) et Alcheringa, The Eternal Dreamtime (Austral Books, Sydney, 2008).

\section{BIBLIOGRAPHIE DES VILLEMINOT}

Villeminot Jacques et Betty, 1961. Chez les Papous, Paris, Hachette.

—, 1964. Coutumes et mours des Papous, Paris, SCEMI.

—, 1967. Nowvelle-Guinée, Paris, Marabout Université.

—, 1967. Australie, Terre de fortune, Paris, Robert Laffont.

—, 1967. Les seigneurs des mers du Sud, Paris, Robert Laffont.

—, 1975. Australie sauvage, Paris, Presses de la Cité.

-, 1979. Nouvelle-Guinée, les Papous chasseurs de têtes, Paris, Presse de la Cité.

—, 1983. Australie, la grande barrière de corail, Paris, Presse de la Cité.

—, 1987. L'Ouest australien, Paris, Solar.

—, 1993. Au commencement des temps, l'Australie, Paris, A. Barthélemy.

-, 2010. Les hommes oubliés d'Océanie, Paris, Glénat.

Villeminot Betty, 1979. La petite fille des mers $d u$ Sud, Paris, G.P.

—, 1979. Zap le petit Papou, Paris, G.P.

—, 1997. Alcheringa, Le temps du rêve éternel, $\mathrm{Pa}$ ris, Albin Michel.

-, 1998. El Tiempo del Sueno eterno, Barcelone, B.S.A.

—, 2004. Alcheringa, Sydney, Duffy et Snellgrove.

-,2008. Alcheringa, The Eternal Dreamtime, Sydney, Austral Books.

Villeminot Jacques, 1954. Boomerang (2 volumes), Paris, Julliard.

—, 1959. Paradis primitifs, Paris, Hachette.

-, 2008. Mémoire d'Océanie, vivre de son rêve, Paris, Pages du monde.

\section{PRINCIPAUX FILMS DES VILLEMINOT}

Jacques et Betty Villeminot ont réalisé des films en $16 \mathrm{~mm}$ et en $35 \mathrm{~mm}$, présentés dans le cadre des conférences « Connaissance du Monde » et sur les chaînes de télévision. Plusieurs d'entre eux ont été primés dans des festivals internationaux.

Villeminot Jacques et Betty, 1951. Areyonga.

—, 1951. Visages d'Océanie.

-, 1958. Paradis primitif.

—, 1960. Les Hommes oubliés.

—, 1963. Les Hommes de la Waghi.

-, 1965. Les Hommes du Sepik.

—, 1969. Les seigneurs des mers du Sud.

-, 1970. Visages mystérieux d'Océanie.

-, 1970. Troglodytes modernes.

—, 1976. Capture des buffles et des dromadaires.

-, 1976. Un homme, deux fermmes et des pythons.

-, 1977. Aux sources de l'art.

—, 1978. Asmat, les hommes arbres.

\section{BIBLIOGRAPHIE}

Bounoure Gilles, 2011. Compte rendu de l'ouvrage de Jacques et Betty Villeminot, Les hommes oubliés d'Océanie (2010), Journal de la Société des Océanistes 132, pp. 189-190.

CoIffier Christian, 2009. Sculpture faîtière, Village de Kanganaman, in Yves Le Fur, $M u$ sée du quai Branly, La Collection, Paris, Skira Flammarion/musée du quai Branly, pp. 228229.

-, 2010. Compte rendu de l'ouvrage d'Eva Raabe, Reisen und Entdecken. Vom Sepik an den Main, Hintergründe einer Ausstellung (2008), Journal de la Société des Océanistes 131, pp. 264-266.

Coupaye Ludovic, 2009. Faire l'expérience de l'espace: la frise tëkët des Abelam, Arts \& Cultures, Genève, Association des Amis des Musées Barbier-Mueller, pp. 210-225.

Dousset Laurent, 2011. Mythes, missiles et cannibales, Le récit d'un premier contact en Australie, Paris, Société des Océanistes, Publication de la Société des Océanistes 50. 